\title{
A Moment-Based Approach for Deskewing Rotationally Symmetric Shapes
}

\author{
Soo-Chang Pei and Ji-Hwei Horng \\ Department of Electrical Engineering \\ National Taiwan University \\ Taipei, Taiwan, R.O.C. \\ Email: pei@cc.ee.ntu.edu.tw
}

\begin{abstract}
The covariance matrix of a rotationally symmtric shape is a scalar identity matrix. In this paper, we apply this property of covariance matrix to deskew the skewed shape of rotational symmetry. The parameters of the deskew transformation matrix are solved by letting the covariance matrix of the transformed shape be equal to a scalar identity matrix. Then, the rotationally symmetric shape can be recovered by the deskew transformation matrix. The method does not rely on continuous contours, since only second-order moments of the input shape are required to be computed. Experimental results are also presented.
\end{abstract}

\section{Introduction}

Many researchers on the area of image analysis have pay attention to the importance of rotational symmtry property of planer shapes. Lots of methods have been proposed to normalize the rotationally symmetric shapes (abbreviated as RSS henceforth) [1][5]. However, none of them has dealt with the skewed shape of rotational symmetry.

In real applications, the view direction is not usually perpendicular to the plane containing RSS, it results in a skewed RSS on the image plane. Thus, the complete normalization procedure must include the deskewing stage.

The term skewed symmetry was first defined by Kanade [6].

"It means a class of 2-D shapes in which the symmetry is found along lines not necessarily perpendic- ular to the axis, but at a fixed angle to it." A more detailed explaination is given by Friedberg [7].

"Skew symmetric figures are planar figures in the image space that are generated from planar symmetric figures by either of two processes:

1. rotation in three dimensions followed by orthographic projection onto the image plane;

2. oblique coordinate transformation followed by rotation in the image plane."

The term symmetry in these definitions refers to reflective symmtry. We can define the skewed rotional symmtry by modifying the definition given by Friedberg.

Difinition. Skewed rotionally symmetric shapes are planar shapes in the image space that are generated from planar rotationally symmetric shapes by either of two processes:

1. rotation in three dimensions followed by orthographic projection onto the image plane;

2. oblique coordinate transformation followed by rotation in the image plane.

The problem of deskewing reflective symmetry and deskewing rotational symmetry are somewhat similar. In fact, the set of reflective symmetric shapes overlaps with the set of rotationally symmetric shapes. The relation of these two sets are shown in Figure 1, where a typical shape is given for each subset. Higher-order reflective symmetric shapes[8] are rotationally symmetric shapes. However, not all of the RSS are reflective 
symmetric shapes, an example is shown in Figure 1.

In this paper, the previous work in the area of analyzing the skewed shapes of reflective symmetry is discussed. Then, a moment-based method for deskewing the skewed RSS is proposed. Several experimental results are also presented.

\section{Previous work}

Before proposing the method for deskewing the skewed RSS, let us review previous work in the area of analyzing skewed shapes of reflective symmtry. The Kanade's work in solving the gradient ambiguity is introduced[6], where the problem of analyzing skewed symmetry was first presented. Then, two momentbased methods for finding the axis of skewed symmetry are discussed[7][8].

Kanade solved the gradient of the plane includes the reflectively symmetric shape. Of course, the threedimensional process is chosen as the model of skew generation process. In his work, the skewed-symmetry axis and the skewed-transverse axis are assumed to be given. For an assumed gradient, the symmetry axis and the transvers axis can be recovered by using the skewed-symmetry axis, the skewed-transverse axis, and the gradient. The gradient is solved by the equation that the inner product of the symmetry axis and the transverse axis is equal to zero, since they are perpendicular to each other. However, there is still a trouble that it results in an infinite number of possible solutions, that is, there is an ambiquity in determining the gradient. Kanade suggested that the least slanted gradient may be the most reasonable selection. Notice that, he solved the gradient ambiquity but did not give a method for finding the skewed-symmetry axis and the skewed-transverse axis.

Friedberg proposed a moment-based method to find the parameters $\alpha$ and $\beta$ of the deskew coordinate transformation matrix. The two-dimensional process is chosen as the model of skew generation process. He used the property that the covariance matrix for a symmetric shape is a diagonal matrix, that is, the moment $m_{11}$ is necessarily equal to zero. For a set of assumed parameters $\alpha$ and $\beta$ of the deskew coordinate transformation matrix, the moment $m_{11}$ of the deskewed shape can be written as a function of the set of parameters and the second-order moments of the skewed shape. Let the moment $m_{11}$ of the deskewed shape be equal to zero, resulting in a constraint on the set of parameters $\alpha$ and $\beta$. Stated another way, $\alpha$ can be described as a function of $\beta$ and vice versa. The constraint reduces the search space from a two-dimensional parameter space to a onedimensional parameter space. The sector symmetry evaluator is applied to search desired solutions along the one-dimensional parameter space. This method is computationally expensive, since a search process is required. In addition, it does not guarantee to get exact solutions.

Gross also chose the two-dimensional process as the model of skew generation process. He decomposed the skew transformation matrix into a shear matrix followed by a rotation matrix. Therefore, the deskewing process was decomposed into two steps: the recovering of rotation and the recovering of shear. The rotation parameter $\alpha$ is solved by letting the moments of inverse rotated shape satisfy the constraint $n_{1,1} n_{0, n}=$ $n_{0,2} n_{1, n-1}$, where $n$ is the degree of symmtry. Then, the shear parameter $\beta$ is solved by substituting the moments of the inverse rotated shape into the equation $\cot \beta=n_{1,1} / n_{0,2}$. This method assumes that the degree of symmetry is given, which is not usually the case in real applications. For higher-order symmetries, numeric methods need to be used and the computational complexity is increased. Furthermore, this method is not applicable to deskew the skewed RSS, since the RSS may not have a axis of symmetry and the constraints $n_{\text {odd }, j}=0$ and $n_{1,1} n_{0, n}=n_{0,2} n_{1, n-1}$ are no longer valid.

\section{Proposed method}

\section{A. Constraints}

Let us assume that the given shape $S_{q}=\left\{q_{k} \mid k=\right.$ $1,2, \ldots, n$.$\} is a skewed version of the \operatorname{RSS} S_{p}=$ $\left\{p_{k} \mid k=1,2, \ldots, n\right.$. $\}$. All coordinates of the image points are measured relative to the centroid of the shape, that is, translation invariance is assumed. The $(\mathrm{i}, \mathrm{j})$ moment of $S_{p}$ is defined by $m_{i j}=\sum_{k} x_{k}^{i} y_{k}^{j}$, where $\left[x_{k}, y_{k}\right]^{T}=p_{k}$. The covariance matrix of the RSS $S_{p}$ is defined by

$$
M=\sum_{k} p_{k} p_{k}^{T}=\left[\begin{array}{ll}
m_{20} & m_{11} \\
m_{11} & m_{02}
\end{array}\right] .
$$

It can be proved[1] that the covariance matrix of an RSS is a scalar identity matrix. That is, there are two constraints on the RSS $m_{11}=0$ and $m_{20}-m_{02}=$ 0 . In comparison with the method proposed by Friedberg[7], an additional constraint $m_{20}-m_{02}=0$ is available, since the given shape is an RSS instead 
of a reflectively symmetric shape. Of course, the solution space to be searched is reduced by the additional constraint.

\section{B. Solution}

In this paper, the two-dimensional process is chosen as the model of skew generation process. Since the given shape $S_{q}$ is a skewed version of the RSS $S_{p}$, they can be related by $q_{k}=T p_{k}, k=1,2, \ldots, n$, where $T$ is the skew transformation matrix and is defined by

$$
T=\left[\begin{array}{cc}
\cos \alpha & \cos \alpha \cot \beta-\sin \alpha \\
\sin \alpha & \sin \alpha \cot \beta+\cos \alpha
\end{array}\right], \begin{aligned}
& 0 \leq \alpha<\pi \\
& 0<\beta<\pi
\end{aligned}
$$

The angles $\alpha$ and $\beta$ are the degrees of rotation and shear, respectively, in the skew generation process. Then, the covariance matrices of the RSS $M$ and the skewed RSS $N$ are related by

$$
N=\sum_{k} q_{k} q_{k}^{T}=\sum_{k}\left(T p_{k}\right)\left(T p_{k}\right)^{T}=T M T^{T} .
$$

It can be rewritten as

$$
M=T^{-1} N T^{-T} .
$$

After manipulations, the two constraints can be written as

$$
\begin{array}{r}
{\left[n_{20}^{2}-n_{20} n_{02}+n_{11}^{2}\right] \tan ^{4} \alpha+\left[-4 n_{20} n_{11}\right] \tan ^{3} \alpha} \\
+\left[6 n_{11}^{2}\right] \tan ^{2} \alpha+\left[-4 n_{11} n_{02}\right] \tan \alpha \\
+\left[-n_{20} n_{02}+n_{11}^{2}+n_{02}^{2}\right]=0 \\
{\left[-n_{11} \tan ^{2} \alpha-\left(n_{20}-n_{02}\right) \tan \alpha+n_{11}\right] \tan \beta} \\
+\left[-n_{20} \tan ^{2} \alpha+2 n_{11} \tan \alpha-n_{02}\right]=0 .
\end{array}
$$

It can be proved that solving the univariate quartic polynomial, in terms of $\tan \alpha$, in Equation (2) gives exactly two distinct real roots. Substituting the two roots into Equation (3), there exists exactly one solution such that $0<\beta<\pi / 2$.

\section{Algorithm}

We propose the deskew algorithm as follows

1. Compute the covariance matrix $N$ of the given skewed $\operatorname{RSS} S_{q}=\left\{q_{k} \mid k=1,2, \ldots, n.\right\}$.

$$
N=\sum_{k=1}^{n} q_{k} q_{k}^{T}
$$

2. Compute the two distinct real solutions of the following quartic equation in terms of $\tan \alpha$ by using explicit formula.

$$
\begin{array}{r}
{\left[n_{20}^{2}-n_{20} n_{02}+n_{11}^{2}\right] \tan ^{4} \alpha} \\
+\left[-4 n_{20} n_{11}\right] \tan ^{3} \alpha \\
+\left[6 n_{11}^{2}\right] \tan ^{2} \alpha+\left[-4 n_{11} n_{02}\right] \tan \alpha \\
+\left[-n_{20} n_{02}+n_{11}^{2}+n_{02}^{2}\right]=0 .
\end{array}
$$

3. For each resulting $\tan \alpha$, solve its corresponding $\tan \beta$ by substituting into the following equation.

$$
\begin{array}{r}
{\left[-n_{11} \tan ^{2} \alpha-\left(n_{20}-n_{02}\right) \tan \alpha+n_{11}\right] \tan \beta} \\
+\left[-n_{20} \tan ^{2} \alpha+2 n_{11} \tan \alpha-n_{02}\right]=0 .
\end{array}
$$

4. Apply the pair of parameters containing the $\beta$ within $(0, \pi / 2)$, which is unique, to construct the deskew transformation matrix $T^{-1}$.

$$
T^{-1}=\left[\begin{array}{cc}
\cos \alpha+\sin \alpha \cot \beta & \sin \alpha-\cos \alpha \cot \beta \\
-\sin \alpha & \cos \alpha
\end{array}\right] .
$$

5. Deskew the given skewed RSS by using the deskew transformation matrix.

$$
p_{k}=T^{-1} q_{k}, k=1,2, \ldots, n .
$$

\section{Experimental results}

The algorithm is programmed in MATLAB lauguage and executed by workstation. The CPU time required is within one second for each case. In fact, it is the fastest moment-based algorithm for deskewing the skewed RSS. Because once the moments of the skewed shape are achieved, the parameters of the deskew transformation matrix can be computed directly by applying the explicit formula.

The input skewed RSS of our first experiment is shown in Figure 2(a). To understand how we get the solution, the loci of parameters corresponding to our two constraints are plotted in Figure 2(b). Each point on a locus represents a possible solution under its corresponding constriant. The loci are plotted by equally sampling on the $\alpha$ space and solving $\beta$ for each given value of $\alpha$. The locus corresponding to the constraint $m_{11}=0$ is represented by solid line and the locus corresponding the constraint $m_{20}-m_{02}=0$ is represented by crosses. Because the constraint $m_{20}-m_{02}=0$ is a quadratic equation in $\cot \beta$, there 
are two $\beta$ values for each $\alpha$ value or is no solution sometimes(when the two $\cot \beta$ values are complex conjugates). The intersections of the two loci are solutions of the problem. The two deskewed shapes corresponding to the two intersections are shown in Figure 2(c) and (d). In fact, applying our algorithm gives the version shown in Figure 2(c). The shape of Figure 2(d) is a rotated version of Figure 2(c) and can be ignored.

An additional experiment is shown in Figure 3. Notice that, the deskewed shape shown in Figure $3(\mathrm{~b})$ is a RSS and also a reflectively symmetric shape(higher-order symmetry[8]).

\section{Conclusion}

In this paper, we propose an $\mathcal{O}(n)$ algorithm to deskew the skewed RSS. The moment-based algorithm does not rely on smooth or continuous contours. We do not intend to finding the axis of symmetry, because a given RSS may not have any axis of reflective symmetry. However, it does have axes, several kinds of axes are proposed by previous researchers[1]-[4].

The experimental result confirms our derivations of constraints and shows availability of our algorithm. The algorithm gives accurate estimation of the skew parameters $\alpha$ and $\beta$.

After applying our algorithm, any of the algorithms proposed in [1]-[5] can be used to normalize the deskewed RSS and thus the whole normalization procedure is completed.

\section{References}

[1] Wen-Hsiang Tsai and Sheng-Lin Chou, Detection of generalized principal axes in rotationally symmetric shapes, Pattern Recognition, Vol. 24, No. 2, pp. 95-104, 1991.

[2] Soo-Chang Pei and Chao-Nan Lin, Normalization of rotationally symmetric shapes for pattern recognition, Pattern Recognition. Vol. 25, No. 9, pp. 913-920, 1992.

[3] Ja-Chen Lin, Universal principal axes: an easy-toconstruct tool useful in defining shape orientations for almost every kind of shape, Pattern. Recognition, Vol. 26, No. 4, pp. 485-493, 1993.

[4] Ja-Chen Lin, Wen-Hsiang Tsai, and Jun-Ann Chen, Detecting number of folds by a simple mathematical property, Pattern Recognition Letters 15, pp. 1081-1088, 1994.
[5] Raymond K.K. Yip, Wilson C.Y. Lam, Peter K.S. Tam, and Dennis N.K. Leung, A Hough transform technique for the detection of rotational symmetry, Pattern Recognition Letters 15, pp. 919-928, 1994.

[6] Takeo Kanade, Recovery of the three-dimensional shape of an object from a single view, Artificial Intelligence 17, pp. 409-460, 1981.

[7] Stuart A. Friedberg, Finding Axes of Skewed Symmetry, Computer Vision, Graphics, and Image Processing 34, pp. 138-155, 1986.

[8] Ari D. Gross and Terrance E. Boult, Analyzing Skewed Symmetries, International Joumal of Computer Vision, pp. 91-111, 1994.

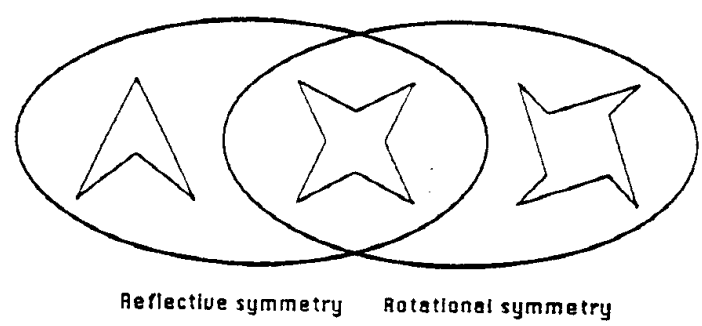

Figure t The relas:on of reflecive symmetry and rotacional symmst.-

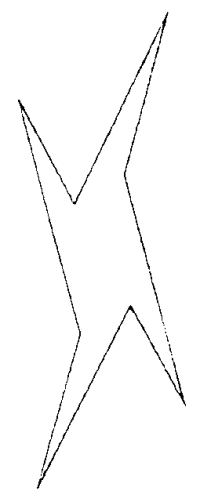

Figure 2(a) The skewed version of a rotaticnally symmetric snape. 


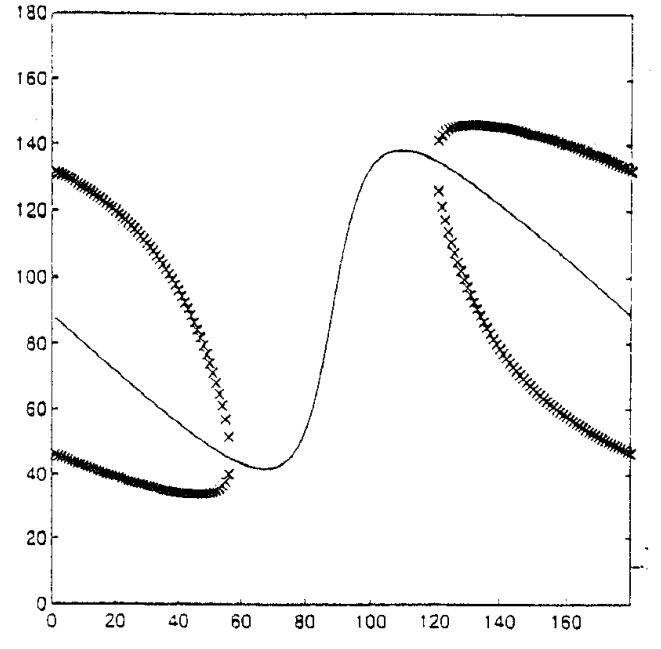

Figure 2(b) The loci of our constraints. The locus corresponding to the constraint $m_{11}=0$ is represented by solid line and the locus corresponding to the constraint $\mathbb{m}_{20}-\pi_{0 z}=0$ is represented by crosses.

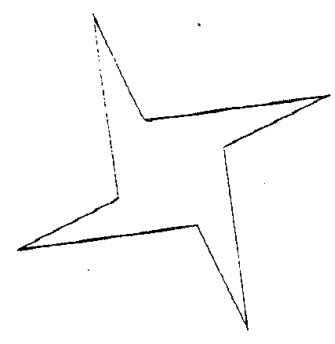

Figure 2(c) The deskewed version of the shape shown in (a).

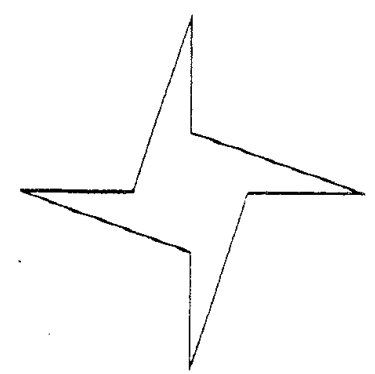

Figure 2(d) The deskewed version of the shape shown in (a). It is a rotated version of (c).

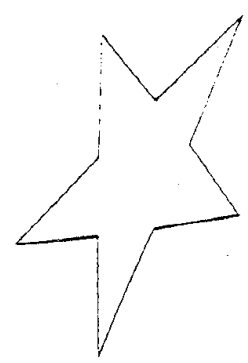

Figure $3(a)$ The skewed version of a rotationally symmetric shape It is atso a reflectively symmetric shape.

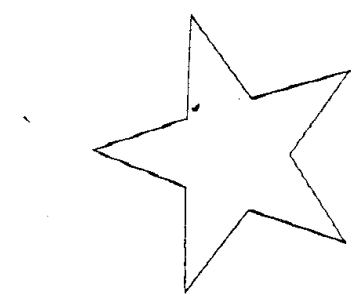

Figure $3(b)$ The deskewed version of the shape shown in (a). 